\title{
LRRK2 Kinase Activity Induces Mitochondrial Fission in Microglia via Drp1 and Modulates Neuroinflammation
}

\author{
Dong Hwan $\mathrm{Ho}^{1 \dagger}$, A Reum Je ${ }^{2 \dagger}$, Haejin Lee ${ }^{2}$, Ilhong Son ${ }^{1,3}$, Hee-Seok Kweon ${ }^{2 *}$, \\ Hyung-Gun Kim ${ }^{4 *}$ and Wongi Seol ${ }^{1 *}$ \\ ${ }^{1}$ InAm Neuroscience Research Center, Sanbon Medical Center, College of Medicine, Wonkwang University, Gunpo 15865, \\ ${ }^{2}$ Electron Microscopy Research Center, Korea Basic Science Institute (KBSI), Daejeon 34133, \\ ${ }^{3}$ Department of Neurology, Sanbon Medical Center, College of Medicine, Wonkwang University, Gunpo 15865, \\ ${ }^{4}$ Department of Pharmacology, College of Medicine, Dankook University, Cheonan 31116, Korea
}

\begin{abstract}
Leucine-rich repeat kinase 2 (LRRK2) mutations are the most common genetic cause of Parkinsons disease (PD). LRRK2 contains a functional kinase domain and G2019S, the most prevalent LRRK2 pathogenic mutation, increases its kinase activity. LRRK2 regulates mitochondria morphology and autophagy in neurons. LPS treatment increases LRRK2 protein level and mitochondrial fission in microglia, and down-regulation of LRRK2 expression or inhibition of its kinase activity attenuates microglia activation. Here, we evaluated the direct role of LRRK2 G2019S in mitochondrial dynamics in microglia. Initial observation of microglia in G2019S transgenic mice revealed a decrease in mitochondrial area and shortage of microglial processes compared with their littermates. Next, we elucidated the molecular mechanisms of these phenotypes. Treatment of BV2 cells and primary microglia with LPS enhanced mitochondrial fission and increased Drp1, a mitochondrial fission marker, as previously reported. Importantly, both phenotypes were rescued by treatment with GSK2578215A, a LRRK2 kinase inhibitor. Finally, the protein levels of CD68, an active microglia marker, Drpl and TNF- $a$ were significantly higher in brain lysates of G2019S transgenic mice compared with the levels in their littermates. Taken together, our data suggest that LRRK2 could promote microglial mitochondrial alteration via Drp1 in a kinase-dependent manner, resulting in stimulation of pro-inflammatory responses. This mechanism in microglia might be a potential target to develop PD therapy since neuroinflammation by active microglia is a major symptom of PD.
\end{abstract}

Key words: Parkinson's disease, LRRK2, microglia, neuroinflammation, mitochondrial fission, Drp1

Received June 1,2018, Revised June 28,2018,

Accepted June 29, 2018

* To whom correspondence should be addressed.

Hee-Seok Kweon, TEL: 82-43-240-5440, FAX: 82-42-865-3939

e-mail: hskweon@kbsi.re.kr

Hyung-Gun Kim, TEL: 82-41-550-3867, FAX: 82-41-551-3866

e-mail:hgkimm@dankook.ac.kr

Wongi Seol, TEL: 82-31-390-2411, FAX: 82-31-890-2414

e-mail:wseolha@gmail.com

TThese authors contributed equally.

\section{INTRODUCTION}

Leucine rich-repeat kinase 2 (LRRK2) is a causal gene of Parkinson's disease (PD, $[1,2])$, which is the second most common neurodegenerative disease. LRRK2 contains functional kinase and GTPase domains. G2019S, the most prevalent LRRK2 familial pathogenic mutant, is known to exhibit increased kinase activity [3]. Most investigations into the pathogenic mechanism of LRRK2 
have been focused on neuronal functions because PD is neurodegenerative disease. The studies revealed that LRRK2 regulates vesicle trafficking, autophagy, oxidative stress, neuronal toxicity and mitochondrial dynamics [4-12]. However, neuroinflammation with activated microglia has also been implicated in the pathogenesis of neurodegenerative disorders, such as PD, Alzheimer's disease, Huntington's disease, amyotrophic lateral sclerosis and fronto-temporal dementia [13]. LRRK2 expression in microglia is lower than in dopaminergic neurons [14]. However, protein levels of total LRRK2 and active phospho-LRRK2 were significantly increased by LPS treatment activating microglia in vivo or in vitro [15]. Previous studies reported that inhibition of LRRK2 kinase activity or decreased expression of LRRK2 in microglia attenuated the pro-inflammatory response [15-17] whereas increased kinase activity of G2019S promotes inflammation [18]. Our previous study also suggested that p53 phosphorylation by LRRK2 contributed to tumor necrosis factor-a (TNFa) release in microglia, resulting in decreased neuronal survival [19].

Mitochondria dysfunction is widely accepted as one of the pathogenic mechanisms of PD [20]. In fact, other genetic causes of PD such as mutations in Parkin and PINK1 are known to impair mitochondria $[21,22]$. Other studies also suggested that LRRK2 regulated mitochondrial dynamics and affected mitochondria damages in neuronal cells [12, 23-25]. Drp1, also known as mitochondrial fission protein Dlp1, is a component of the LRRK2 interactome and is responsible for LRRK2-mediated mitochondria fission in neurons [12]. However, no study examined the effect of LRRK2 on mitochondrial morphology in microglia. Interestingly, LPS stimulations of microglia also increased mitochondrial fission, Drpl level, production of pro-inflammatory cytokines and ROS as well as kinase activity of LRRK2 [15, 16, 26-28]. In addition, phagocytic activity of macrophages is regulated by Drp1mediated mitochondrial fission [29]. These results suggest that LRRK2 facilitated neuroinflammatory response in microglia by regulating mitochondrial dynamics.

\section{MATERIALS AND METHODS}

\section{Antibodies}

The following antibodies were used: LRRK2 (N241A/34, NeuroMabs, 75-253), pS935-LRRK2 (UDD210(12), Abcam, 133450), Drp1 (C5, Santa Cruz, 271583), Tom 20 (FL-145, Santa Cruz, sc11415), CD68 (3F103, Santa Cruz, sc70761), TNFa (52B83, Santa Cruz, sc-52746), Iba-1 (Wako, 019-19741), GFAP (Sigma-Aldrich, G3839), $\beta$-actin (Santa Cruz, sc-47778), $\alpha$-tubulin (DM1A, Sigma, T9026), horseradish peroxidase-conjugated goat anti-rabbit or anti-mouse IgG (Jackson ImmunoResearch. 111-035-003 or 115-
035-003) and goat anti-rabbit or anti-mouse IgG H\&L (Alexa Fluor $^{\circledR}$ 594, Invitrogen, ab150080 or Alexa Fluor ${ }^{\circledR} 488$, Invitrogen, ab150113).

\section{Cell culture and TNF $\alpha$ enzyme-linked immunosorbent as- say (ELISA)}

BV2 murine microglia cells $\left(4 \times 10^{5}\right)$ were seeded in $35 \mathrm{~mm}$ dishes. Next day, the cells were treated with GSK2578215A (hereinafter GSK, $1 \mu \mathrm{M}$, Torcris Bioscience) for $30 \mathrm{~min}$ followed by LPS ( $1 \mu \mathrm{g} /$ $\mathrm{ml}$ ) treatment for $6 \mathrm{~h}$. Then, the cells was fixed with $4 \%$ paraformaldehyde (PFA, Wako) for immunofluorescence analysis or harvested for Western blot analysis. Culture media derived from these BV2 cells and rat primary microglia administrated with the same treatment as BV2 cells, and brain lysates from G2019S transgenic mouse or littermate were used to perform mouse TNFa ELISA (Biolegend) or rat TNFa DuoSet ELISA (R\&D systems) according to the sample species and the manufacturer's instruction.

\section{Handling mouse and mouse brain preparation}

G2019S transgenic (TG) mice [strain B6; C3-Tg (PDGFBLRRK2*G2019S) 340D jmo/J, stock number 016575, The Jackson Laboratory, [30]] were housed in a specific pathogen-free facility at Dankook University Animal Facility on a 12:12-h light/dark cycle. Animal experiments were approved by the Institutional Animal Care and Use Committee of Dankook University (DKU16-035). Animals were provided free access to food and water. TG mice and their normal control littermates were sacrificed by cervical dislocation. Brains were lysed with 1\% Triton X-100 and protease inhibitor cocktail (Calbiochem) in phosphate buffered saline (PBS). Lysates were homogenized 10 times using a 17-gauge needle, cooled on ice for $30 \mathrm{~min}$, and centrifuged at 4,000 $\times \mathrm{g}$ for $10 \mathrm{~min}$ at $4^{\circ} \mathrm{C}$. Each supernatant was collected and analyzed by Western blot.

\section{Preparation and culture of microglia}

Rat primary microglial cells were isolated from neonatal rats in the plage as previously described [31].

\section{Transmission electron microscope (TEM)}

G2019S TG and non-TG mice were anesthetized with pentobarbital and transcardially perfused with PBS and 4\% PFA. Striatum was then dissected from each brain and subjected to EM processing as described previously [32]. Tissues were fixed in $2.5 \%$ glutaraldehyde in $0.1 \mathrm{M}$ phosphate buffer ( $\mathrm{pH} 7.4$ ) for $2 \mathrm{~h}$ at $4^{\circ} \mathrm{C}$ and post-fixed with $1 \%$ osmium tetroxide on ice for $2 \mathrm{~h}$. Tissues were then embedded in Epon 812 after dehydration in an ethanol and propylene oxide series. Polymerization was conducted using pure 
resin at $70^{\circ} \mathrm{C}$ for two days. Ultrathin sections (70 $\mathrm{nm}$ in thickness) were obtained with an ultramicrotome (UltraCut-UCT, Leica, Austria) and collected on 100-mesh copper grids. After staining with $2 \%$ uranyl acetate and lead citrate, the sections were examined by transmission electron microcopy (TEM) (Technai G ${ }^{2}$ Spirit TWIN, FEI, USA) at $120 \mathrm{kV}$. To quantify mitochondrial number and size, electron micrographs were obtained at a magnification of $8,000 \times$ and analyzed blindly using Image J software.

\section{Immunofluorescence, confocal microscopy and quantita- tive analysis of mitochondria morphology}

BV2 cells were treated as indicated, fixed and permeablized with $0.1 \%$ Triton X-100 in DPBS for 5 min at RT. After 1 h of blocking with $3 \%$ BSA and $1 \%$ goat serum in DPBS, cells were incubated with Tom 20 antibodies in blocking solution for $8 \mathrm{~h}$ at $4^{\circ} \mathrm{C}$ followed by incubation with Alexa Fluoro 594 secondary antibodies in blocking solution at RT for $2 \mathrm{~h}$ with shaking. Hoechst (Thermo Scientific) staining was accomplished during the final DPBS washing step. Cells on inverted cover slip were mounted using ProlongGold (Invitrogen). The experiments were repeated in triplicate. Each set of experiments was observed under a Zeiss LS55 confocal microscope and more than five images were randomly obtained in Airyscan mode. In total, 10 12 cells were selected and their mitochondria ( $n=16 \sim 242 /$ cell, total 412 1094) were analyzed. To determine the mitochondria area and elongation, mitochondrial morphology was measured using the Mito-Morphology Macro of Image J software as previously described $[33,34]$. The RFP channel of cells stained with Tom 20 was extracted and converted to 8-bit grayscale, and then inverted. Individual mitochondria were highlighted on the images and analyzed for changes in mitochondrial area and circularity. The mitochondrial circularity was inversed to calculate mitochondrial elongation [34].

\section{Immunochemistry}

LRRK2 TG and non-TG mice were anesthetized as described above. Their brains were post-fixed in 4\% PFA and coronally sliced into $200 \mu \mathrm{m}$ sections using a vibratome (VT1000A, Leica, Austria). Sections were washed with PBS and treated with 3\% hydrogen peroxide to block endogenous peroxidase activity. Sections were then incubated overnight with anti-Ibal (1:500, Waco) followed by treatment with biotinylated anti-rabbit antibody (1:200, Vector Laboratories). Anti-Ibal antibody was detected by avidin-biotinperoxidase complex (Vector Laboratories) followed by incubation with nickel-enhanced 3, 3 ' diaminobenzidine (DAB, Sigma). To determine the morphological characteristics of Ibal-labeled microglia, images were obtained at $40 \times$ objective magnification using an inverted light microscope (CRX41, Olympus, Japan). The process number and length were analyzed manually using Image J software by an unbiased operator.

\section{Western blot analysis and densitometry}

Harvested sample were loaded on to $11 \%$ SDS-PAGE gel or $4 \sim 15 \%$ gradient pre-cast gel (Bio-Rad) followed by transfer onto nitrocellulose membranes. The remaining steps and densitometric analysis were similar to the methods described previously [35].

\section{Statistical analysis}

Data derived from Western blots, ELISA, measured mitochondrial fission, and microglial processes were analyzed and imaged with Prism 6.0 (GraphPad). Data are expressed as means \pm standard error of the mean (SEM). Statistical analyses of data are described in each figure legend.

\section{RESULTS}

\section{Increased mitochondrial fission and microglial activation in G2019 transgenic mouse brain}

To investigate whether LRRK2 regulates mitochondrial fission in microglia, we compared the ultrastructure of mitochondria in the microglia of striatum in G2019S TG and control non-TG littermate mice. We confirmed that the mitochondria number was significantly increased in TG, although the total mitochondria area did not change significantly (Fig. 1A 1C). To analyze fission rates in microglia, we compared the average mitochondria areas between TG and control littermates. The average mitochondria area of TG microglia was significantly decreased compared with the non-TG control (Fig. 1D). Previous studies demonstrated shortening and decreased microglial processes in active microglia $[36,37]$. We observed similar phenotypes (decreased numbers and shortened length of microglial processes) of TG compared with their non-TG littermates (Fig. 2). These results support the notion that G2019S LRRK2 mutation induced microglial activation and mitochondrial fission in microglia in vivo.

\section{Treatment with LRRK2 kinase inhibitor reversed mito- chondrial fission induced by LPS-mediated LRRK2 kinase activation}

Previous studies revealed that treatment with ligands in microglial activation increased LRRK2 kinase activity in the primary microglia or murine BV2 microglia cell line $[15,38]$. Furthermore, increased mitochondrial fission by LPS in BV2 was demonstrated in a previous study [26]. To determine whether LRRK2 kinase activity directly affected mitochondrial fission in LPSstimulated microglia, BV2 cells were treated with LPS alone or 
A

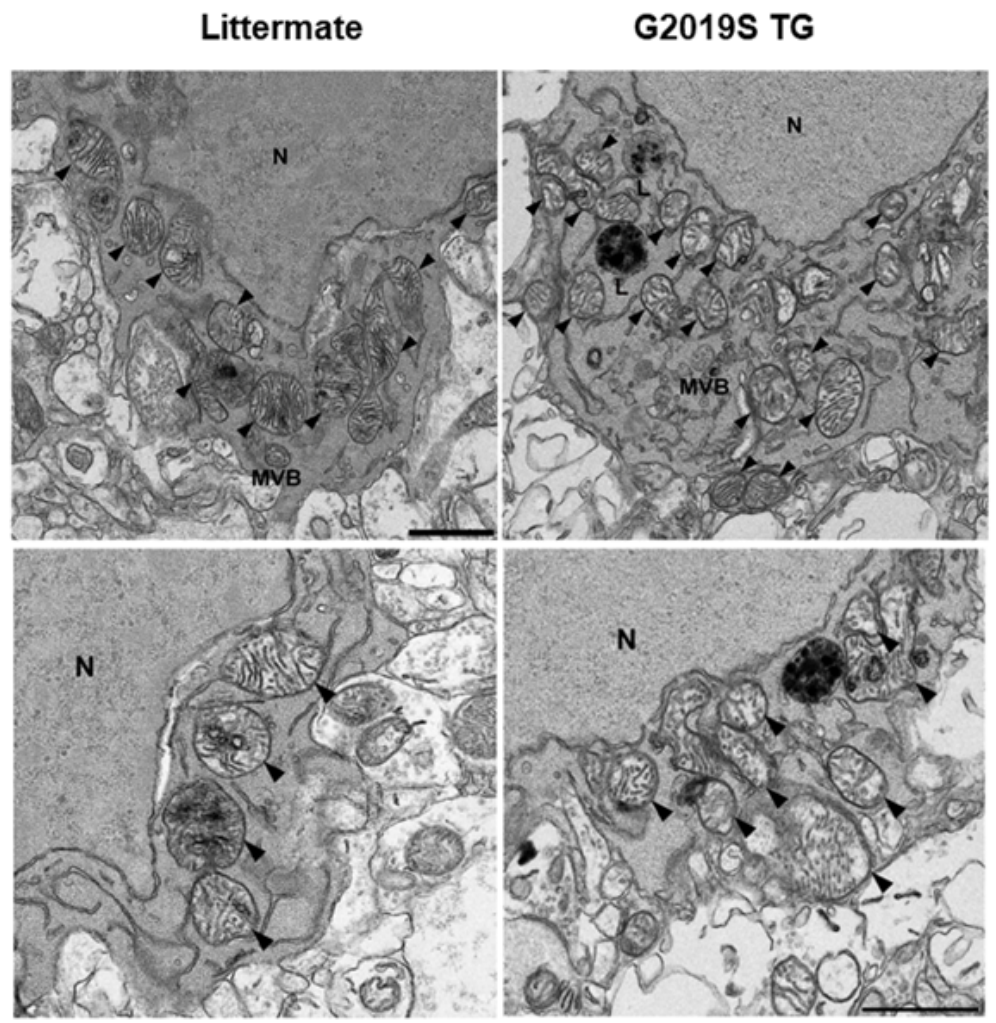

B
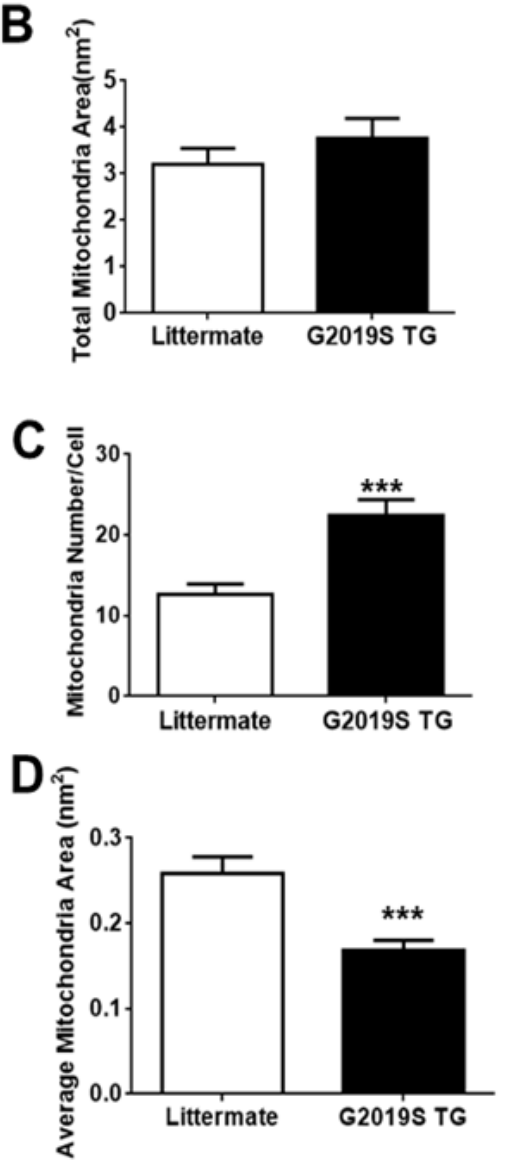

Fig. 1. Ultrastructural analyses of mitochondria in microglia in the striatum of G2019S TG and non-TG littermate mice (14-month, n=3). (A) Ultrastructure of mitochondria in microglia obtained using TEM. Cells were blindly selected and the mitochondrial area was measured. The number of observed cells and total measured mitochondria $\left(\mathrm{nm}^{2}\right)$ were 15 and 190 for wild-type littermates and 14 and 314 for G2019S-TG, respectively. The arrowheads indicate mitochondria used for analysis. N, nucleus; L, Lysosome; MVB, multivesicular body; Scale bar=1 $\mu \mathrm{m}$. Graphic analyses of total mitochondria area/cell (B) and average mitochondria number/cell (C) are shown. Average individual mitochondrial area, total mitochondrial area/cell divided by mitochondria number/cell, are also shown (D). Statistical analysis was performed by Student's $t$-test, ${ }^{* *} \mathrm{p}<0.001$.

LPS and GSK2578215A (GSK, LRRK2 kinase inhibitor), and their mitochondrial morphologies were examined using an immunofluorescence assay. Image analysis revealed that LPS treatment significantly decreased mitochondrial elongation, suggesting reduced mitochondrial length [34], whereas co-treatment of LPS and GSK significantly diminished the difference (Fig. 3A, B). In addition, LPS treatment reduced mitochondrial area, and co-treatment abrogated the reduction, although without any statistical significance (Fig. 3A, C). The shortening of mitochondria in the LPS-treated BV2 cells is similar to the phenotype observed in neuronal soma in the G2019S TG [30]. These results suggest that LRRK2 regulated mitochondrial morphology in microglia as well as in neuron [30].

Previous studies reported that LRRK2 regulated Drp1 protein level in neurons [12], which prompted us to evaluate Drpl level in microglia. Both BV2 (Fig. 4A 4F) and rat primary microglia cells (Fig. 4G 4L) were treated with LPS alone or LPS and GSK, followed by analysis of Drp1 and Tom 20 protein levels. LPS treatment significantly increased the Drpl level compared with the sample containing vehicle alone or with co-treatment with LPS and GSK whereas the level of Tom20, a mitochondria marker, was relatively similar among samples in both cell types (Fig. 4). The ratio of Drp1 level to Tom20 (Drp1/Tom20) was increased by LPS treatment compared with other samples and, as expected, the difference was more apparent in primary microglia than BV2 cells (Fig. 4E, K). Absence of significant changes in Tom 20 levels suggested that the total mass of mitochondria was not significantly altered by either LPS or LPS/GSK treatment (Fig. 4D, J). However, the increased fission rate induced by LPS was reversed by co-treatment with GSK. The western blot with phospho-S935 LRRK2 antibody confirmed inhibition of LRRK2 kinase by GSK as reported 
A

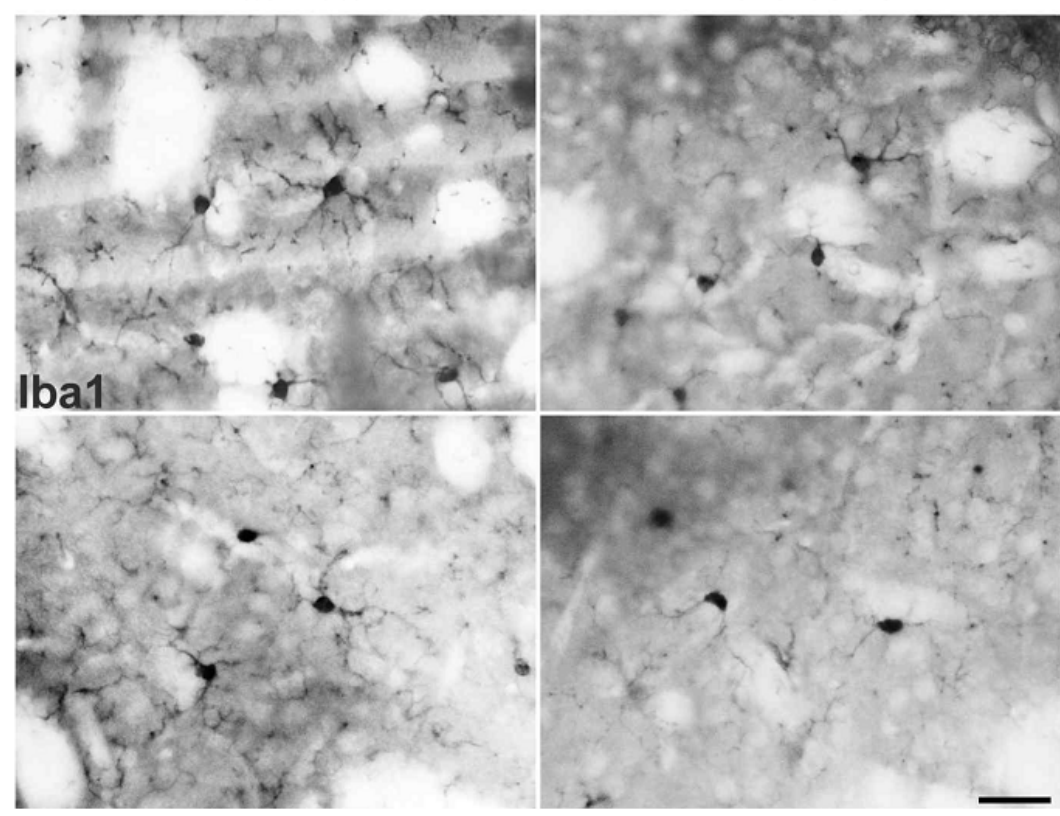

B

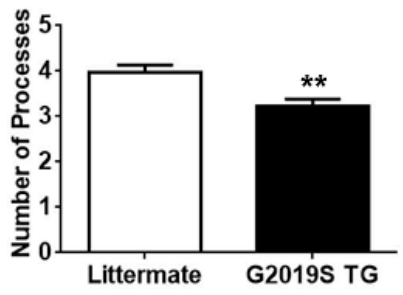

C

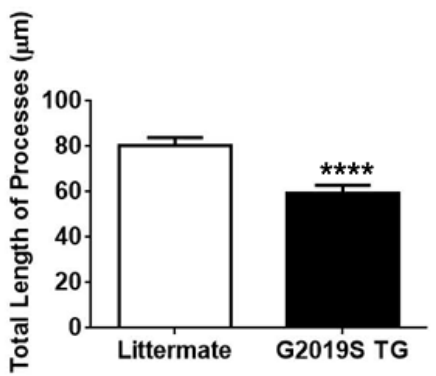

Fig. 2. Morphological analyses of activated microglia in the striatum of G2019S TG and non-TG littermate mice. (A) Images of Iba1-positive microglia captured via inverted light microscopy. Their process numbers and lengths were analyzed ( $n=3)$. A total of 60 and 61 cells were analyzed for wild-type littermate and G2019S-TG, respectively. Scale bar $=50 \mu \mathrm{m}$. Numbers (B) and lengths (C) of Iba1-labeled microglia processes in different groups are shown. Student's $t$-test was used for statistical analysis, ${ }^{* *} \mathrm{p}<0.01,{ }^{* * *} \mathrm{p}<0.0001$.

A
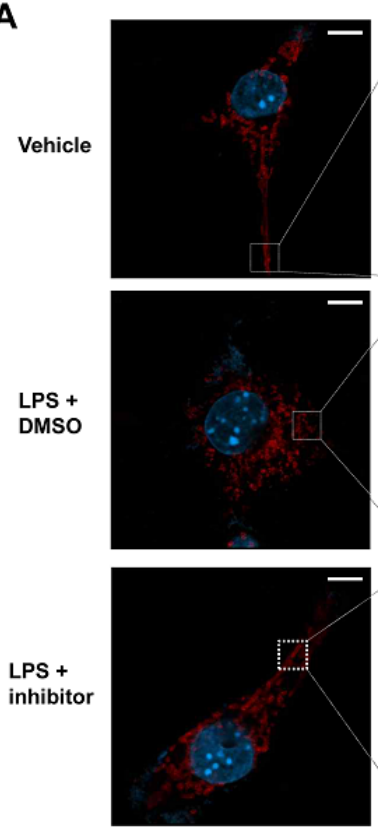
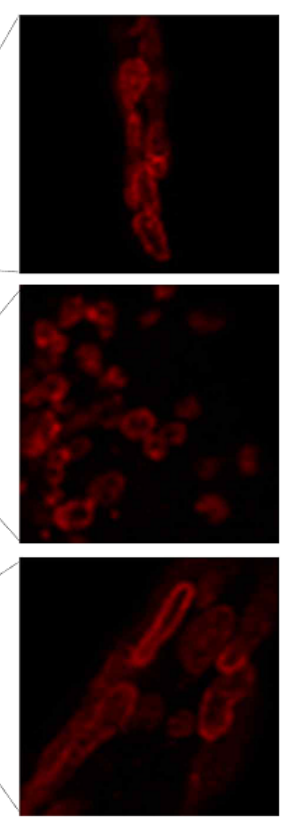

B
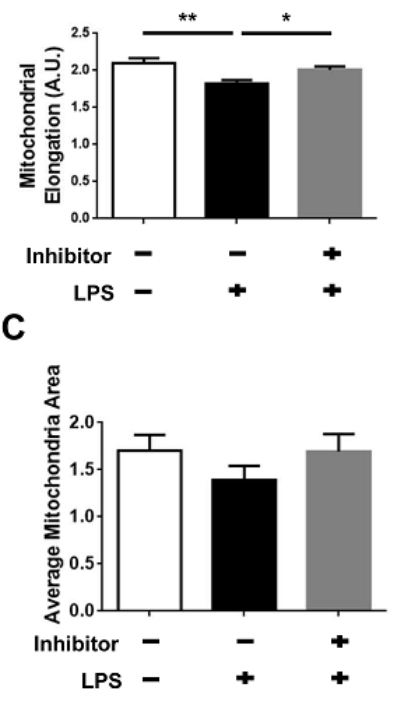

Fig. 3. LRRK2 kinase inhibitor ameliorated LPS-induced mitochondrial fission in BV2. (A) LPS $(1 \mu \mathrm{g} / \mathrm{ml}$ ) or vehicle (water) was used for $6 \mathrm{~h}$ treatment following $30 \mathrm{~min}$ pre-treatment of GSK $(1 \mu \mathrm{M}$, LRRK2 kinase inhibitor) or vehicle (DMSO). Images of Tom 20 (red) and Hoechst (blue)-stained BV2 cells were obtained using the Airyscan mode of the LS55 confocal microscope. Scale bar=10 $\mu \mathrm{m}(\mathrm{n}=3$, number of cells $=10 \sim 11$, number of mitochondria measured $=412 \sim 1042)$. Both elongation (B) and area (C) of mitochondria were measured using the Image J program. The statistical analysis was performed by one-way analysis of variance (ANOVA) with Tukey's post-hoc test. previously (Fig. 4B, H, [38]). We also tested whether LPS mediated cytokine secretion to confirm activation of BV2 cells and primary microglia by LPS. LPS-mediated TNF- $\alpha$ secretion was significantly increased and the increase was almost restored by GSK pretreatment in both cell types (Fig. 4F, L). These data demonstrate that mitochondrial fission up-regulated by LPS stimulation was attenuated by the inhibitor of LRRK2 kinase.

\section{Up-regulated neuroinflammatory responses and mito-} chondrial fission associated with aging in G2019S-TG mice

We consolidated the evidence underlying G2019S-mediated neuroinflammation and mitochondrial fission in microglia in vivo 
A

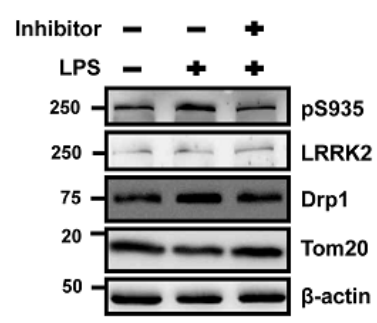

B

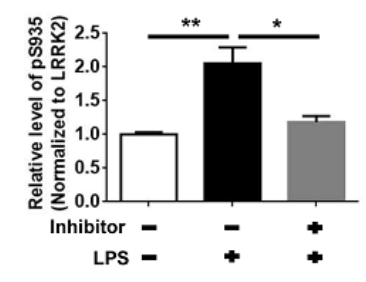

C

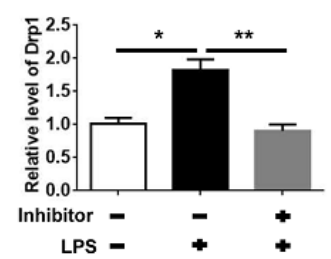

D

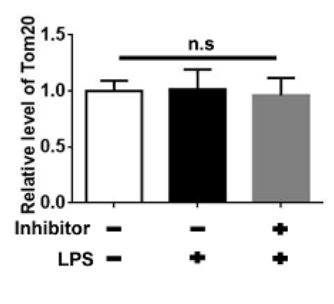

E

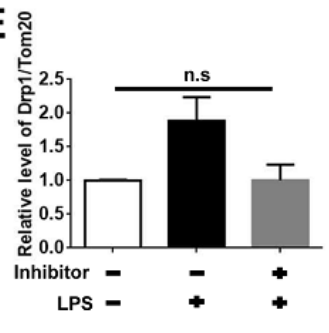

$\mathbf{F}$

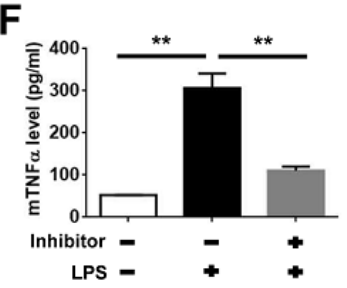

G

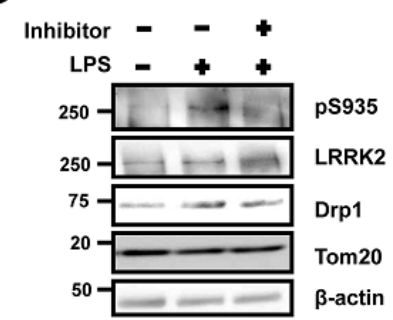

H

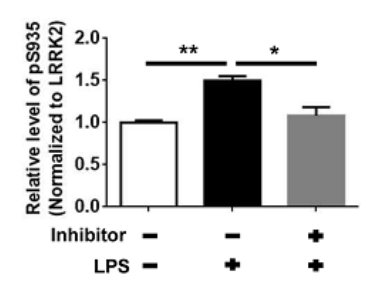

I

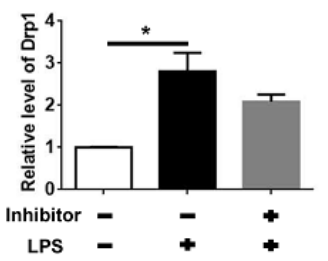

J

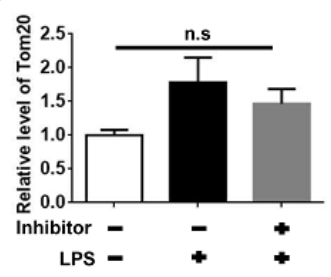

$\mathrm{K}$ 。

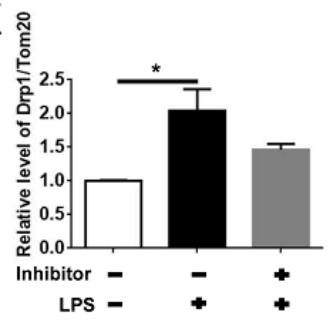

L

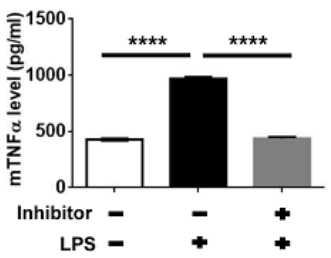

Fig. 4. LRRK2 kinase inhibitor (GSK) treatment abolished Drp1 increase and TNF-a release induced by LPS treatment. BV2 (A) and primary rat microglia $(\mathrm{G})$ cells were treated as described in Fig. 3. Analysis and a representative of three Western blot images are shown. Effectiveness of GSK treatment was confirmed by decrease in LRRK2 pS935 autophosphorylation level (pS935/total LRRK2, B \&H). Relative densitometric analyses of Drp1 (C \& I) or Tom 20 (D \& J), normalized by $\beta$-actin, and Drp1/Tom 20 (E \& K) levels in BV2 (C $\sim$ E) and primary rat microglia (H L) cells are shown. The secretion of TNF- $\alpha$ by murine BV2 cells (F) and rat primary microglia (L) was measured by ELISA. The statistical analysis was performed by ANOVA with Bonferroni post-hoc test. ${ }^{*} \mathrm{p}<0.05,{ }^{* *} \mathrm{p}<0.01,{ }^{* * * *} \mathrm{p}<0.0001$, n.s., not significant.

using old (age $>52$ weeks) and young (18 26 weeks) G2019S TG and non-TG littermates. Brain lysates of these mice were analyzed by the indicated antibodies after SDS-PAGE and ELISA (Fig. 5). Previous studies showed that the expression of CD 68 is a pivotal marker of activated microglia [39]. Strikingly, CD68 levels were increased at 5.0-fold and 7.8-fold in both young and old G2019STG, respectively, compared with non-TG littermates, although the increase was not statistically significant in old G2019S-TG (Fig. 5A, B). Significant increases in Drp1 and Drp1/Tom20 levels were only observed in old G2019S-TG, although young G2019S-TG mice showed a slight increase in Drp1/Tom20 (Fig. 5A, 5C 5E). Furthermore, there was no significant difference in Tom20 (Fig. 5E). Both young and old G2019S-TG mice exhibited significantly increased levels of soluble TNFa compared with age-matched non-TG littermates (Fig. 5F), supporting increase of CD68 in both young and old TG mice (Fig. 5B). In summary, the TG data in Fig. 5 suggest that G2019S LRRK2 gradually increased mitochondrial fission associated with aging via accumulation of neuroinflammatory responses, including activated microglia and induced cyto- kines initiated at a relatively early age.

\section{DISCUSSION}

Neurodegenerative diseases are often associated with mitochondrial dysfunction and microglial activation. In fact, PD patients show highly activated microglia in substantia nigra and enhanced pro-inflammatory cytokine expression $[40,41]$.

Numerous studies have shown that mitochondrial dysfunction is critical in PD pathogenesis [20, 22, 42]. Several pathogenic mutations of PD causal genes such as Parkin, PINK-1, DJ-1, and LRRK2 are known to dysregulate mitochondrial function [22]. Treatment of BV2 with rotenone, which is a PD-causing toxin, activated microglia and generated cytokines and ROS [43] although the phenotypes were not observed in primary microglia [44]. LPS treatment also increased LRRK2 kinase activity in the brains of mice expressing FLAG-LRRK2, and treatment with LRRK2 kinase inhibitor or shLRRK2 ameliorated TNFa release [15]. Therefore, we tested whether LRRK2 regulated microglial mitochondrial 

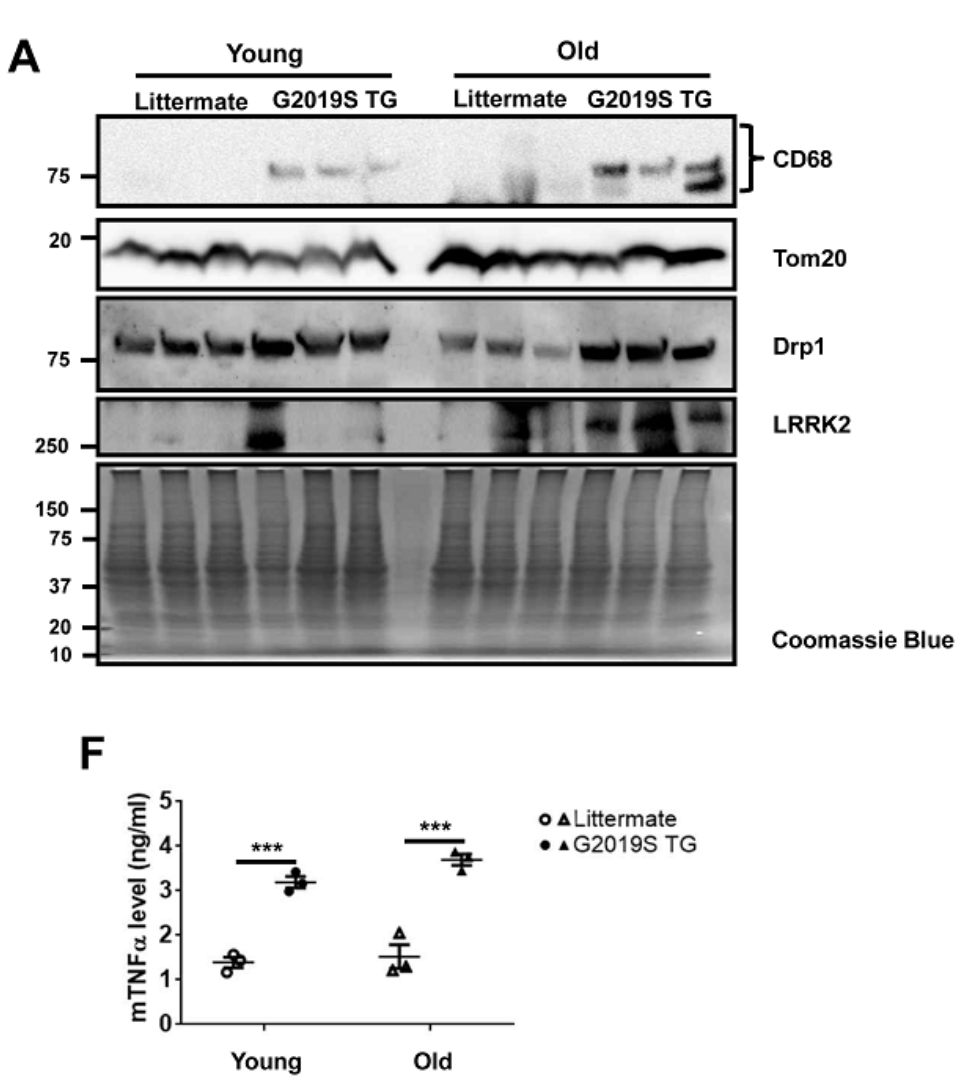

B

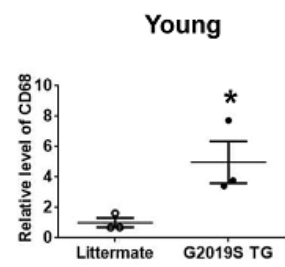

C

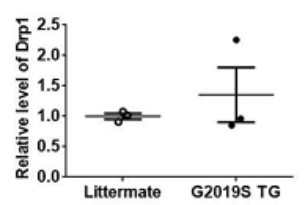

D

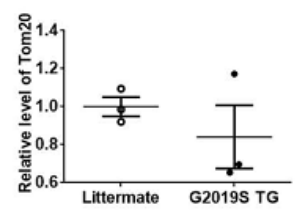

E

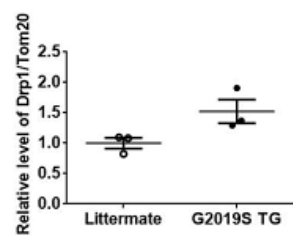

Old
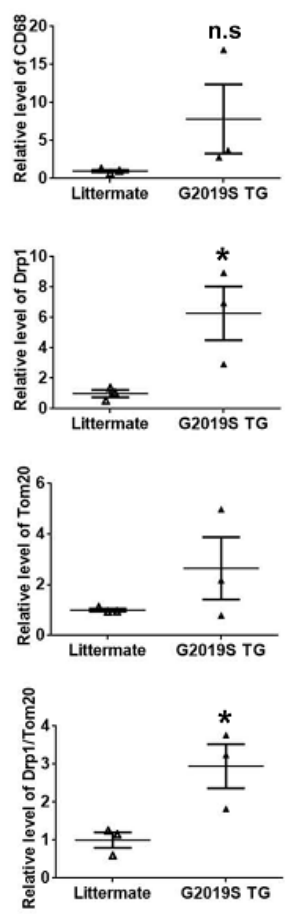

Fig. 5. Enhanced neuroinflammatory responses and increased mitochondrial fission protein in G2019S TG mouse lysates. Both young (4 6 months, $\mathrm{n}=3$ ) and old (12 19 months, n=3) G2019S TG mice and non-TG littermates were used. (A) Brain lysates were subjected to Western blot using the indicated antibodies. Bands of interest were normalized by Coomassie Blue staining. Graphic analysis of each Western blot is shown. Student's $t$-test was used to separately compare the relative levels of CD68 (B), Drp1 (C), Tom20 (D) and Drp1/Tom20 (E) between non-TG littermate and G2019S TG in young and old mice. (F) The TNF- $\alpha$ level of brain lysates was measured by ELISA. The statistical analysis was performed by ANOVA with Bonferroni post-hoc test. ${ }^{*} \mathrm{p}<0.05,{ }^{* * *} \mathrm{p}<0.001$, n.s., not significant.

function in vivo, primary microglia and BV2 cells. Our study reveals that LRRK2 kinase activity promotes mitochondrial fission via increase of Drpl and triggers a pro-inflammatory response in microglia of G2019S TG mice brains (Fig. 1, 2), BV2 cells (Fig. 3 5) and rat primary microglia (Fig. 4).

Drpl is a critical mediator of neuroinflammatory response [26]. LPS treatment of microglia increased mitochondrial fission, Drp1 protein level and ROS production in BV2 and mouse primary microglia [26,27]. Moreover, down-regulation of Drp1 by shDrp1 reduced mitochondrial fragmentation, cytokine production and ROS production in BV2 [26].

Our study connects LRRK2 activation and Drpl activation, two discrete intermediate steps in LPS-mediated neuroinflammation, and suggests a signal transduction pathway from LPS to neuroinflammation via LRRK2-meiated Drp1 activation in microglia. An earlier study reported that LRRK2 phosphorylates Drp1 at T595 and the expression of T595A Drp1 corrects G2019S-mediated mitochondrial fission in neurons [45] whereas another report sug- gested LRRK2-mediated phosphorylation of Drp1 at S616 [46]. In addition, inhibition of Drpl reported to protect neurons in dopaminergic system in an MPTP-mediated PD model [47], providing additional evidence for toxic mechanism of LRRK2 G2019S. Because functions of phosphorylated Drpl are various depending on cell types and phosphorylation sites [48], it is necessary to investigate the effect of LRRK2-mediated phosphorylation of Drp1 on mitochondrial fission in microglia in a future study.

The G2019S TG mouse we used has been originally reported to express G2019S in a neuron-specific manner because the gene was under the CMV E-PDGF- $\beta$ promoter $[30,49]$. The original report to study expression of luciferase under the same PDGF- $\beta$ promoter showed that at least $15 \%$ of cells expressing luciferase are not neurons [49], leaving a possibility for direct expression of G2019S in microglia of the TG mice. Moreover, neuronal LRRK2 expression increases secretion of $a$-synuclein [50] which is able to activate microglia in a paracrine manner [51]. Previous studies reported that damaged neurons activated microglia in vitro $[52$, 

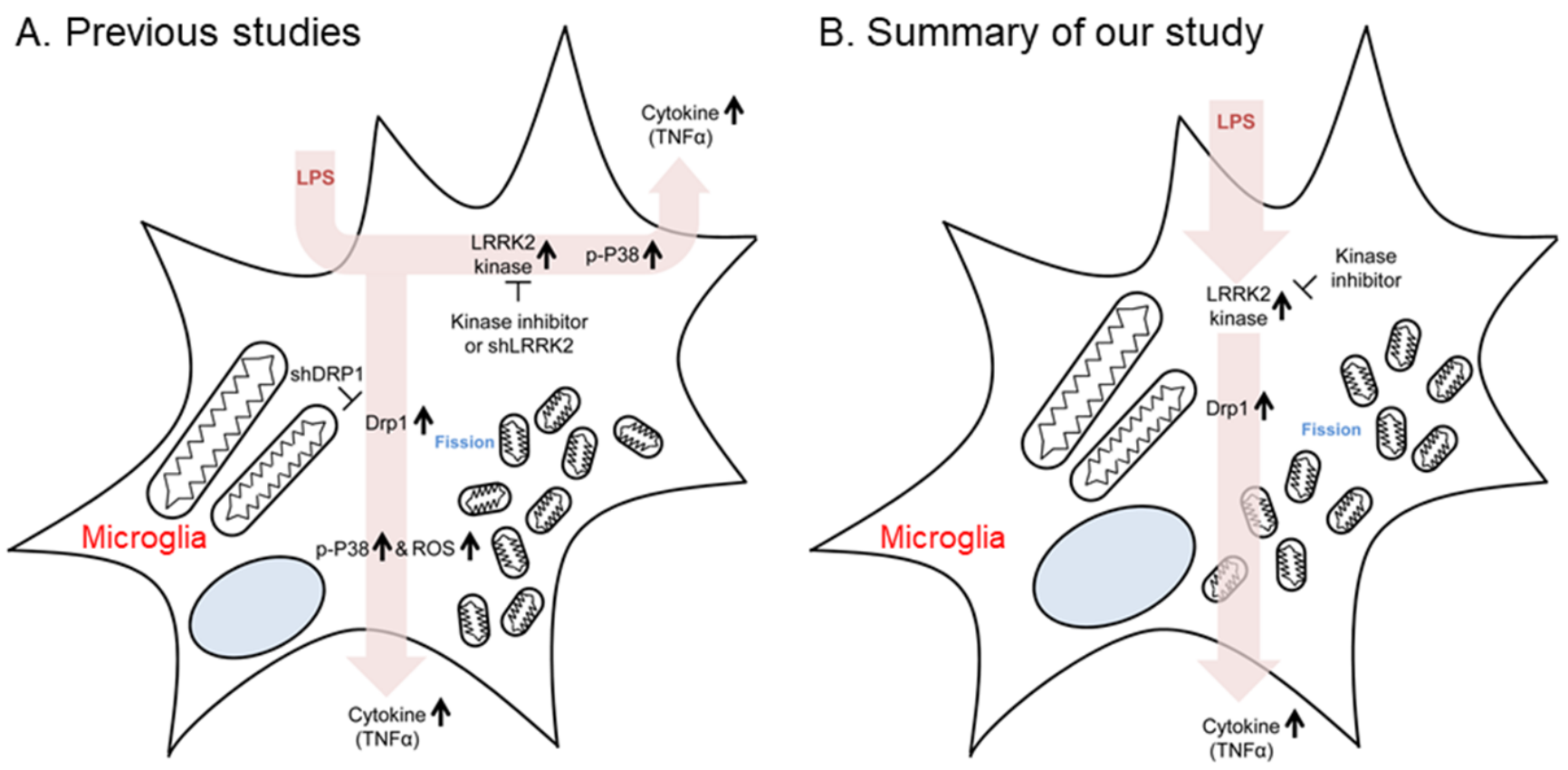

Fig. 6. A summary of our study. (A) A summary based on the previous studies $[12,15,16,26]$. (B) Our study summary.

53]. Therefore, neuronal expression of G2019S in TG mice brains is possible to activate nearby microglia and affects mitochondria morphology in those microglia.

We have previously reported that LRRK2 phosphorylates p53 at T304 and T377 residues and the expression of phosphomimic p53 in BV2 cells induces TNFa expression and its release, resulting in neuronal death [19]. The combined evidence derived from our study suggests that G2019S LRRK2 contributed to the progression of PD by altering the physical condition of mitochondria in microglia during the neuroinflammation. In addition, we suggest Drp1 as a missing link between LPS-induced LRRK2 activation and mitochondrial dynamics, both of which have been reported to induce microglial activation $[15,16,26]$. Furthermore, our data which was summarized in Fig. 6 suggest that LRRK2 kinase inhibitor attenuates pro-inflammatory response by rescuing of mitochondrial dysregulation in microglia. Therefore, Drpl regulation by LRRK2 kinase in microglia may be one of the pathogenic mechanisms in PD mediated by LRRK2 G2019S mutation, indicating LRRK2 kinase as a promising PD therapeutic target.

\section{ACKNOWLEDGEMENTS}

We thank our team members for their assistance. This study was supported by a grant from InAm Neuroscience Research Center (Sanbon Hospital, Wonkwang University, Korea to WS \& IS) and grants awarded by the Basic Science Research Program (2015R1C1A2A01051755 to WS), Korea Basic Science Institute
(T38210 to HSK) through the National Research Foundation (NRF) funded by the Ministry of Education, Science and Technology, Republic of Korea.

\section{REFERENCES}

1. Paisán-Ruíz C, Jain S, Evans EW, Gilks WP, Simón J, van der Brug M, López de Munain A, Aparicio S, Gil AM, Khan N, Johnson J, Martinez JR, Nicholl D, Carrera IM, Pena AS, de Silva R, Lees A, Martí-Massó JF, Pérez-Tur J, Wood NW, Singleton $\mathrm{AB}$ (2004) Cloning of the gene containing mutations that cause PARK8-linked Parkinson's disease. Neuron 44:595-600.

2. Zimprich A, Biskup S, Leitner P, Lichtner P, Farrer M, Lincoln S, Kachergus J, Hulihan M, Uitti RJ, Calne DB, Stoessl AJ, Pfeiffer RF, Patenge N, Carbajal IC, Vieregge P, Asmus F, MüllerMyhsok B, Dickson DW, Meitinger T, Strom TM, Wszolek ZK, Gasser T (2004) Mutations in LRRK2 cause autosomaldominant parkinsonism with pleomorphic pathology. Neuron 44:601-607.

3. West AB, Moore DJ, Biskup S, Bugayenko A, Smith WW, Ross CA, Dawson VL, Dawson TM (2005) Parkinson's diseaseassociated mutations in leucine-rich repeat kinase 2 augment kinase activity. Proc Natl Acad Sci U S A 102:16842-16847.

4. Yun HJ, Park J, Ho DH, Kim H, Kim CH, Oh H, Ga I, Seo H, Chang S, Son I, Seol W (2013) LRRK2 phosphorylates Snapin and inhibits interaction of Snapin with SNAP-25. Exp Mol Med 45:e36. 
5. Shin N, Jeong H, Kwon J, Heo HY, Kwon JJ, Yun HJ, Kim CH, Han BS, Tong Y, Shen J, Hatano T, Hattori N, Kim KS, Chang S, Seol W (2008) LRRK2 regulates synaptic vesicle endocytosis. Exp Cell Res 314:2055-2065.

6. Gómez-Suaga P, Fdez E, Blanca Ramírez M, Hilfiker S (2012) A link between autophagy and the pathophysiology of LRRK2 in Parkinson’s disease. Parkinsons Dis 2012:324521.

7. Manzoni C, Lewis PA (2017) LRRK2 and autophagy. Adv Neurobiol 14:89-105.

8. Nguyen HN, Byers B, Cord B, Shcheglovitov A, Byrne J, Gujar P, Kee K, Schüle B, Dolmetsch RE, Langston W, Palmer TD, Pera RR (2011) LRRK2 mutant iPSC-derived DA neurons demonstrate increased susceptibility to oxidative stress. Cell Stem Cell 8:267-280.

9. Heo HY, Park JM, Kim CH, Han BS, Kim KS, Seol W (2010) LRRK2 enhances oxidative stress-induced neurotoxicity via its kinase activity. Exp Cell Res 316:649-656.

10. Smith WW, Pei Z, Jiang H, Dawson VL, Dawson TM, Ross CA (2006) Kinase activity of mutant LRRK2 mediates neuronal toxicity. Nat Neurosci 9:1231-1233.

11. West AB, Moore DJ, Choi C, Andrabi SA, Li X, Dikeman D, Biskup S, Zhang Z, Lim KL, Dawson VL, Dawson TM (2007) Parkinson's disease-associated mutations in LRRK2 link enhanced GTP-binding and kinase activities to neuronal toxicity. Hum Mol Genet 16:223-232.

12. Wang X, Yan MH, Fujioka H, Liu J, Wilson-Delfosse A, Chen SG, Perry G, Casadesus G, Zhu X (2012) LRRK2 regulates mitochondrial dynamics and function through direct interaction with DLP1. Hum Mol Genet 21:1931-1944.

13. Amor S, Peferoen LA, Vogel DY, Breur M, van der Valk P, Baker D, van Noort JM (2014) Inflammation in neurodegenerative diseases--an update. Immunology 142:151-166.

14. Miklossy J, Arai T, Guo JP, Klegeris A, Yu S, McGeer EG, McGeer PL (2006) LRRK2 expression in normal and pathologic human brain and in human cell lines. J Neuropathol Exp Neurol 65:953-963.

15. Moehle MS, Webber PJ, Tse T, Sukar N, Standaert DG, DeSilva TM, Cowell RM, West AB (2012) LRRK2 inhibition attenuates microglial inflammatory responses. J Neurosci 32:16021611.

16. Kim B, Yang MS, Choi D, Kim JH, Kim HS, Seol W, Choi S, Jou I, Kim EY, Joe EH (2012) Impaired inflammatory responses in murine Lrrk2-knockdown brain microglia. PLoS One 7:e34693.

17. Puccini JM, Marker DF, Fitzgerald T, Barbieri J, Kim CS, Miller-Rhodes P, Lu SM, Dewhurst S, Gelbard HA (2015) Leucine-rich repeat kinase 2 modulates neuroinflammation and neurotoxicity in models of human immunodeficiency virus 1-associated neurocognitive disorders. J Neurosci 35:52715283.

18. Dzamko N, Rowe DB, Halliday GM (2016) Increased peripheral inflammation in asymptomatic leucine-rich repeat kinase 2 mutation carriers. Mov Disord 31:889-897.

19. Ho DH, Seol W, Eun JH, Son IH (2017) Phosphorylation of p53 by LRRK2 induces microglial tumor necrosis factor a-mediated neurotoxicity. Biochem Biophys Res Commun 482:1088-1094.

20. Winklhofer KF, Haass C (2010) Mitochondrial dysfunction in Parkinson’s disease. Biochim Biophys Acta 1802:29-44.

21. Geisler S, Holmström KM, Treis A, Skujat D, Weber SS, Fiesel FC, Kahle PJ, Springer W (2010) The PINK1/Parkin-mediated mitophagy is compromised by PD-associated mutations. Autophagy 6:871-878.

22. Ryan BJ, Hoek S, Fon EA, Wade-Martins R (2015) Mitochondrial dysfunction and mitophagy in Parkinsons: from familial to sporadic disease. Trends Biochem Sci 40:200-210.

23. Yang S, Xia C, Li S, Du L, Zhang L, Hu Y (2014) Mitochondrial dysfunction driven by the LRRK2-mediated pathway is associated with loss of Purkinje cells and motor coordination deficits in diabetic rat model. Cell Death Dis 5:e1217.

24. Howlett EH, Jensen N, Belmonte F, Zafar F, Hu X, Kluss J, Schüle B, Kaufman BA, Greenamyre JT, Sanders LH (2017) LRRK2 G2019S-induced mitochondrial DNA damage is LRRK2 kinase dependent and inhibition restores $\mathrm{mtDNA}$ integrity in Parkinson's disease. Hum Mol Genet 26:4340-4351.

25. Niu J, Yu M, Wang C, Xu Z (2012) Leucine-rich repeat kinase 2 disturbs mitochondrial dynamics via Dynamin-like protein. J Neurochem 122:650-658.

26. Park J, Choi H, Min JS, Park SJ, Kim JH, Park HJ, Kim B, Chae JI, Yim M, Lee DS (2013) Mitochondrial dynamics modulate the expression of pro-inflammatory mediators in microglial cells. J Neurochem 127:221-232.

27. Katoh M, Wu B, Nguyen HB, Thai TQ, Yamasaki R, Lu H, Rietsch AM, Zorlu MM, Shinozaki Y, Saitoh Y, Saitoh S, Sakoh T, Ikenaka K, Koizumi S, Ransohoff RM, Ohno N (2017) Polymorphic regulation of mitochondrial fission and fusion modifies phenotypes of microglia in neuroinflammation. Sci Rep 7:4942.

28. Chae U, Min JS, Lee H, Song KS, Lee HS, Lee HJ, Lee SR, Lee DS (2017) Chrysophanol suppresses pro-inflammatory response in microglia via regulation of Drp1-dependent mitochondrial fission. Immunopharmacol Immunotoxicol 39:268-275.

29. Wang Y, Subramanian M, Yurdagul A Jr, Barbosa-Lorenzi 
VC, Cai B, de Juan-Sanz J, Ryan TA, Nomura M, Maxfield FR, Tabas I (2017) Mitochondrial fission promotes the continued clearance of apoptotic cells by macrophages. Cell 171:331345.e22.

30. Ramonet D, Daher JP, Lin BM, Stafa K, Kim J, Banerjee R, Westerlund M, Pletnikova O, Glauser L, Yang L, Liu Y, Swing DA, Beal MF, Troncoso JC, McCaffery JM, Jenkins NA, Copeland NG, Galter D, Thomas B, Lee MK, Dawson TM, Dawson VL, Moore DJ (2011) Dopaminergic neuronal loss, reduced neurite complexity and autophagic abnormalities in transgenic mice expressing G2019S mutant LRRK2. PLoS One 6:e18568.

31. Lee HJ, Suk JE, Bae EJ, Lee SJ (2008) Clearance and deposition of extracellular alpha-synuclein aggregates in microglia. Biochem Biophys Res Commun 372:423-428.

32. Choi KJ, Kim MJ, Je AR, Jun S, Lee C, Lee E, Jo M, Huh YH, Kweon HS (2014) Three-dimensional analysis of abnormal ultrastructural alteration in mitochondria of hippocampus of APP/PSEN1 transgenic mouse. J Biosci 39:97-105.

33. Xie W, Chung KK (2012) Alpha-synuclein impairs normal dynamics of mitochondria in cell and animal models of Parkinson's disease. J Neurochem 122:404-414.

34. Dagda RK, Cherra SJ 3rd, Kulich SM, Tandon A, Park D, Chu CT (2009) Loss of PINK1 function promotes mitophagy through effects on oxidative stress and mitochondrial fission. J Biol Chem 284:13843-13855.

35. Ho DH, Kim H, Kim J, Sim H, Ahn H, Kim J, Seo H, Chung KC, Park BJ, Son I, Seol W (2015) Leucine-rich repeat kinase 2 (LRRK2) phosphorylates p53 and induces p $21^{\mathrm{WAF} 1 / \mathrm{CIP} 1} \mathrm{ex}-$ pression. Mol Brain 8:54.

36. Hefendehl JK, Neher JJ, Sühs RB, Kohsaka S, Skodras A, Jucker M (2014) Homeostatic and injury-induced microglia behavior in the aging brain. Aging Cell 13:60-69.

37. Kozlowski C, Weimer RM (2012) An automated method to quantify microglia morphology and application to monitor activation state longitudinally in vivo. PLoS One 7:e31814.

38. Marker DF, Puccini JM, Mockus TE, Barbieri J, Lu SM, Gelbard HA (2012) LRRK2 kinase inhibition prevents pathological microglial phagocytosis in response to HIV-1 Tat protein. J Neuroinflammation 9:261.

39. Walker DG, Lue LF (2015) Immune phenotypes of microglia in human neurodegenerative disease: challenges to detecting microglial polarization in human brains. Alzheimers Res Ther 7:56.

40. McGeer PL, Itagaki S, Boyes BE, McGeer EG (1988) Reactive microglia are positive for HLA-DR in the substantia nigra of Parkinson's and Alzheimer's disease brains. Neurology
38:1285-1291.

41. Sawada M, Imamura K, Nagatsu T (2006) Role of cytokines in inflammatory process in Parkinson's disease. J Neural Transm Suppl 70:373-381.

42. Abou-Sleiman PM, Muqit MM, Wood NW (2006) Expanding insights of mitochondrial dysfunction in Parkinson's disease. Nat Rev Neurosci 7:207-219.

43. Gao F, Chen D, Hu Q, Wang G (2013) Rotenone directly induces BV2 cell activation via the p38 MAPK pathway. PLoS One 8:e72046.

44. Klintworth H, Garden G, Xia Z (2009) Rotenone and paraquat do not directly activate microglia or induce inflammatory cytokine release. Neurosci Lett 462:1-5.

45. Su YC, Qi X (2013) Inhibition of excessive mitochondrial fission reduced aberrant autophagy and neuronal damage caused by LRRK2 G2019S mutation. Hum Mol Genet 22:4545-4561.

46. Esteves AR, G-Fernandes M, Santos D, Januário C, Cardoso SM (2015) The upshot of LRRK2 inhibition to Parkinson's disease paradigm. Mol Neurobiol 52:1804-1820.

47. Filichia E, Hoffer B, Qi X, Luo Y (2016) Inhibition of Drp1 mitochondrial translocation provides neural protection in dopaminergic system in a Parkinson's disease model induced by MPTP. Sci Rep 6:32656.

48. Hu C, Huang Y, Li L (2017) Drp1-dependent mitochondrial fission plays critical roles in physiological and pathological progresses in mammals. Int J Mol Sci 18:144.

49. Liu BH, Wang X, Ma YX, Wang S (2004) CMV enhancer/ human PDGF- $\beta$ promoter for neuron-specific transgene expression. Gene Ther 11:52-60.

50. Kondo K, Obitsu S, Teshima R (2011) a-Synuclein aggregation and transmission are enhanced by leucine-rich repeat kinase 2 in human neuroblastoma SH-SY5Y cells. Biol Pharm Bull 34:1078-1083.

51. Kim C, Ho DH, Suk JE, You S, Michael S, Kang J, Joong Lee S, Masliah E, Hwang D, Lee HJ, Lee SJ (2013) Neuron-released oligomeric $\alpha$-synuclein is an endogenous agonist of TLR2 for paracrine activation of microglia. Nat Commun 4:1562.

52. Sudo S, Tanaka J, Toku K, Desaki J, Matsuda S, Arai T, Sakanaka M, Maeda N (1998) Neurons induce the activation of microglial cells in vitro. Exp Neurol 154:499-510.

53. Witting A, Müller P, Herrmann A, Kettenmann H, Nolte C (2000) Phagocytic clearance of apoptotic neurons by Microglia/Brain macrophages in vitro: involvement of lectin-, integrin-, and phosphatidylserine-mediated recognition. J Neurochem 75:1060-1070. 